\title{
C3-BIOECONOMY
}

Circular and Sustainable Bioeconomy

\section{Políticas Públicas y Bioeconomía en Brasil: la estrategia del Agropolo Campinas-Brasil, Agropolo Campinas-Brasil}

\author{
Sérgio Augusto Morais Carbonell ${ }^{1}$, Luís Augusto Barbosa Cortez ${ }^{2}$, Luís Fernando \\ Ceribelli Madi ${ }^{3}$, Lilian Cristina Anefalos ${ }^{1}$, Ricardo Baldassin Junior ${ }^{1}$ y Rodrigo Lima \\ Verde Leal ${ }^{2}$
}

Autor de Correspondencia: cortez@unicamp.br

\section{Resumen:}

Agropolo Campinas-Brasil fue creado en Campinas, Brasil como una plataforma colaborativa entre algunas instituciones públicas (Instituto Agronómico de Campinas-IAC, Universidad Estadual de Campinas-UNICAMP, Instituto de Tecnología de Alimentos-ITAL, Prefectura de Campinas y TECHNO PARK) con la participación de Empresas Privadas y con el objetivo de cambiar la forma de hacer investigación y promover innovaciones tecnológicas en el área de la bioeconomía tropical. Brasil es un gran productor y exportador de alimentos, pero aún no puede agregar valor justo a sus productos. En este sentido, con el apoyo de la Fundación de Apoyo a la Investigación del Estado de São Paulo - FAPESP, y siguiendo el ejemplo de Agropolis International, Montpellier, Francia, se realizó un estudio en áreas consideradas estratégicas en agricultura, salud, alimentación, química verde y bioenergía. Este artículo presenta algunos de los principales resultados destinados a implementar una hoja de ruta para la bioeconomía tropical.

Palabras clave: Agropolo, Brasil, bioeconomía, desarrollo económico

\section{Public Policies in Bioeconomy in Brazil: the strategy of Agropolo Campinas-Brasil, Agropolo Campinas-Brasil}

\author{
Sérgio Augusto Morais Carbonell ${ }^{1}$, Luís Augusto Barbosa Cortez ${ }^{2}$, Luís Fernando \\ Ceribelli Madi ${ }^{3}$, Lilian Cristina Anefalos ${ }^{1}$, Ricardo Baldassin Junior ${ }^{1}$ y Rodrigo Lima \\ Verde Leal ${ }^{2}$
}

\section{Abstract:}

Agropolo Campinas-Brasil was created in Campinas, Brazil as a collaborative platform among public institutions (Agronomic Institute of Campinas-IAC, State University of Campinas-UNICAMP, Institute of Food Technology-ITAL, the Campinas City Hall, and the TECHNO PARK. The main objective was to change how research organized and to promote technological innovations in the bioeconomy tropical. Brazil is a large producer and exporter of food, but still has difficulties to

1 Instituto Agronómico (IAC), Agência Paulista de Tecnologia de Agronegocios (APTA), Av. Barão de Itapura, 1481, CEP 13020-902, Campinas - SP, Brasil

2 Núcleo Interdisciplinario de Planeación Energética (NIPE), Universidade Estadual de Campinas (UNICAMP), Rua Cora Coralina, 330, CEP 13083-896, Campinas - SP, Brasil

3 Instituto de Tecnologia de Alimentos (ITAL), Agência Paulista de Tecnologia do Agronegócio (APTA), Av. Brasil, 2880, CEP 13070-178, Campinas - SP, Brasil

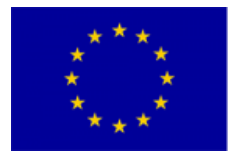

UNIÓN EUROPEA

PROYECTO COFINANCIADO POR EL FONDO EUROPEO DE DESARROL
(EEDER) Una manera de hacer Europa

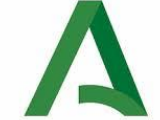

Junta de Andalucía
Andalucía

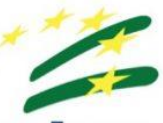

se mueve con Europa 
add value to its products. In this sense, with the support of the Research Foundation of the State of São Paulo - FAPESP, and following the example of Agropolis International, Montpellier, France, a study was carried out in strategic areas in agriculture, health, food, green chemistry, and bioenergy. This article presents some of the main results aimed at implementing a roadmap for the tropical bioeconomy in Brazil.

Keywords: Agropolo, Brazil, bioeconomy, Socioeconomic Development

\section{INTRODUCCIÓN}

Brasil es el país con mayor biodiversidad del planeta y territorialmente el más grande país tropical del mundo. Desde su colonización, ha dependido esencialmente del extractivismo ("pau-brasil4"), de la agricultura (caña-deazúcar y café), y la minería como motor de su economía. En el siglo XX, ha logrado obtener cierta ventaja de los periodos de crisis en Europa y en función de ello, inició -aunque tardíamente-, su proceso de industrialización.

A partir de la década de 70, su agricultura comenzó a mostrar señales de vigor pasando a exportar una gama más variada de productos. Brasil empieza un largo período de desarrollo agroindustrial alcanzando no solamente la autosuficiencia en muchos productos esenciales, sino convirtiéndose en un gran exportador de commodities agrícolas.

En nuestros días, a pesar de la crisis económica de los últimos años, Brasil ha logrado mantenerse como el segundo país exportador más importante de productos agrícolas en el mundo. Esto permite que, a corto plazo, el país sea el que reúna las mejores condiciones para afirmarse como mayor exportador y pueda garantizar el suministro de alimentos para una población cada vez mayor, sobre todo en Asia y África.

No obstante, todo este crecimiento y éxito, Brasil es sobre todo un país que en general no logra obtener suficientes beneficios económicos de sus exportaciones. A pesar de estar entre los primeros productores y exportadores de café, naranja, carnes, soya, maíz; sus productos no son visualizados por su origen y/o marcas brasileñas en los supermercados de países desarrollados. Lo anterior posiblemente se debe a cierta fragilidad de sus empresas locales en

\footnotetext{
4 Pau-Brasil es un árbol con madera roja extraída de ciertas regionales de la Cuesta Brasileira, y utilizado principalmente entre 1500-1600 en Europa para teñir tejidos.
} 
cuanto al comercio exterior, a pesar de las estrategias y esfuerzos del gobierno brasilero, con empresas como la Empresa Brasileña de Investigación Agropecuaria- EMBRAPA.

Para ayudar a superar el problema de la baja valoración de los productos brasileños e instituir una estrategia que genere mayores beneficios económicos, y para aumentar la oferta de las exportaciones con nuevos productos, fue creada la plataforma Agropolo Campinas-Brasil.

El propósito fundamental de esta plataforma es implementar una nueva manera de hacer investigación, más centrada en los productos finales y no en las disciplinas clásicas de investigación. Cuando esto ocurre, los investigadores de diferentes instituciones se relacionan con el objetivo común de superar dificultades, cada uno desde su ámbito de trabajo, cooperando para lograr que un producto final alcance una calidad de excelencia y de sustentabilidad.

\section{2. ¿POR QUÉ LA PLATAFORMA AGROPOLO EN LA CIUDAD DE CAMPINAS, BRASIL?}

Para lograr agregar valor a los "commodities" agrícolas, instituciones tradicionales de investigación de la región de Campinas, retomaron la experiencia de la región de Montpellier, Francia, donde fue creado el Agropolis Internacional (https://www.agropolis.org/).

La ciudad de Campinas, ubicada aproximadamente a $100 \mathrm{Km}$. de São Paulo es el segundo polo económico del estado. Tiene en su historia el "DNA agrícola" en instituciones seculares como el Instituto Agronómico de Campinas. Además, es considerada el tercer mejor ambiente de negocios, investigación e innovación en Brasil (AGENCAMP, 2021). Cuenta también con la Universidad Estadual de Campinas -UNICAMP-, calificada como una de las más importantes por su calidad en investigación y posgrado. La ciudad cuenta también con el Aeropuerto Internacional de Campinas - Viracopos, el más importante en transporte de carga en Brasil. 
Por estas razones, la ciudad de Campinas y sus instituciones agrupan las condiciones adecuadas para reproducir la experiencia exitosa de Montpellier. Fue con este espíritu que se creó el Agropolo Campinas-Brasil en junio 2016.

La plataforma Agropolo Campinas-Brasil (http://www.agropolocampinasbrasil.org/) es una iniciativa de cinco instituciones locales, contando con el apoyo de Agropolis y del Consulado de Francia en São Paulo:

- Instituto Agronómico de Campinas-IAC (www.iac.sp.gov.br),

- Universidad Estadual de Campinas-UNICAMP (www.unicamp.br),

- Instituto de Tecnología de Alimentos-ITAL (www.ital.sp.gov.br),

- $\quad$ Prefectura de Campinas (www.campinas.sp.gov.br), y

- $\quad$ TECHNO PARK Campinas (www.technopark.com.br).

Su objetivo principal se centró en crear una plataforma cooperativa de investigación en agronegocios para el desarrollo de productos de mayor valor agregado a partir de las cadenas productivas existentes.

Figura 1 - El Agropolo Campinas-Brasil y otras plataformas importantes de investigación en agricultura en el mundo.

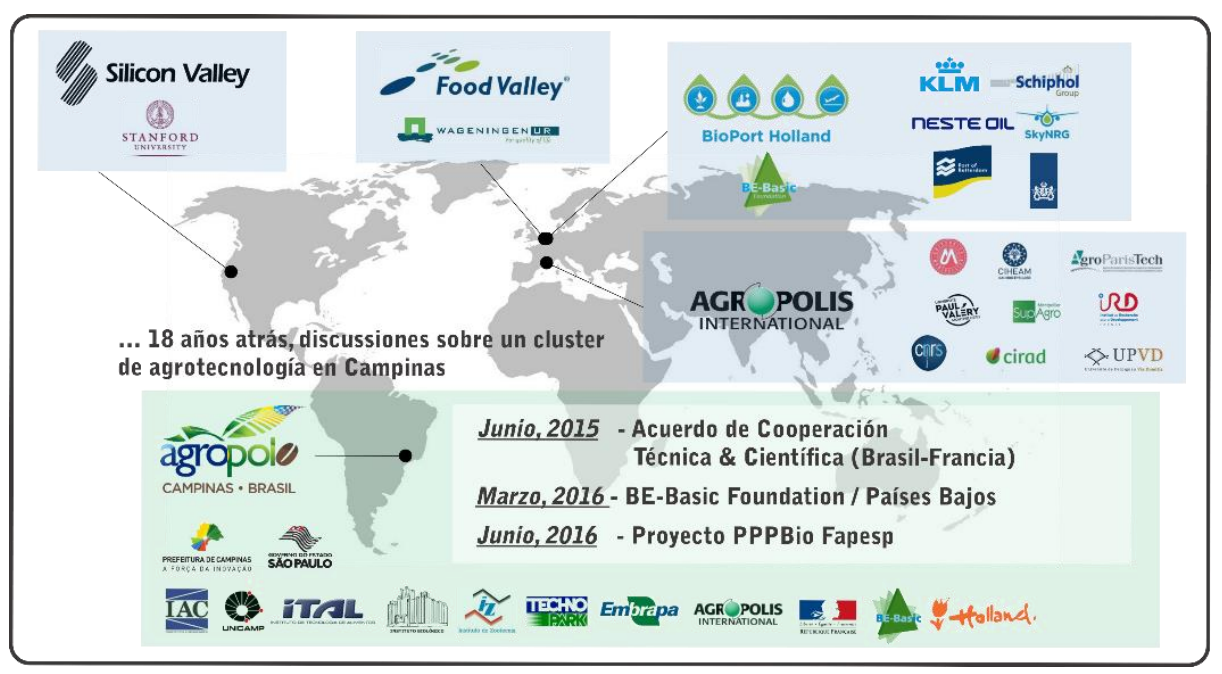

Con financiamiento de la Fundación de Apoyo a la Investigación del Estado de São Paolo-FAPESP, fue creado el primer proyecto Políticas Públicas em Bioeconomía Tropical - PPPBio. con el objetivo de hacer un "roadmap" de bioeconomía tropical. 


\section{EL DIAGNÓSTICO DEL AGROBUSINESS EN BRASIL}

Por diferentes razones puede decirse que el agribusiness en Brasil tiene una historia de muchas dificultades y controversias pero que camina rápidamente para obtener un rol cada vez más importante en la alimentación en el mundo actual y futuro.

Por criterios científicos y tecnológicos se puede afirmar también que la agricultura em Brasil es muy grande y diversa. Hay una agricultura moderna representada por productos como la soya, caña de azúcar, eucalipto, café, y cada vez con mayor importancia, el maíz, naranja, y ciertos productos hortícolas. También en la producción animal existen altos niveles productivos como en la avicultura, cerdos y parte de la ganadería. Sin embargo; en contraposición, todavía existen sectores menos desarrollados, sobre todo en la agricultura familiar y en la producción dedicada al mercado interno. Afortunadamente, este "gap" lentamente está disminuyendo y es posible prever su gradual eliminación a medida que el país se transforme socialmente.

Por otro lado, en los productos donde Brasil presenta un alto grado de competitividad internacional, persisten problemas ligados al bajo valor añadido em su producción. Esto se hace notar cuando se comparan los números absolutos de la exportación total y el de los beneficios financieros obtenidos. Aunque Brasil es actualmente el segundo exportador de productos agropecuarios (el primero son los Estados Unidos), en la renta total obtenida de las exportaciones, el país ocupa el quinto lugar, siendo superado por países como Alemania, Holanda y Francia.

Merecidamente, estos países logran obtener mayor renta debido a la comercialización de una gama de productos innovadores de alto valor, desarrollados a partir de un intenso y dedicado esfuerzo científico y tecnológico, además de recursos humanos altamente calificados y una fuerte interacción público-privada. 
Pero cuando se trata de la agricultura y más específicamente la agricultura tropical, los desafíos son importantes tanto desde el punto de vista de la tierra, la biodiversidad, el agua, como también desde el de los productos tropicales obtenidos, de la falta de experiencia y de recursos humanos calificados para hacer frente a estos desafíos.

Mucho se ha logrado con los institutos y centros de investigación en Brasil. Por ejemplo, la historia del Instituto Agronómico de Campinas - IAC, es una manera de comprender el éxito del café, la caña de azúcar y la naranja en el Estado de São Paulo. Mucho se debe también a la EMBRAPA, con sus trabajos de adaptación de la soja a las condiciones de Brasil.

Sin embargo, todavía existe un largo y difícil camino por recorrer, sobre todo en la atención y aplicación de las demandas por elevar la sustentabilidad y aumentar el valor de la producción. Se espera que el proyecto PPPBio sea parte de esta estrategia.

\section{EL ROADMAP DE BIOECONOMÍA TROPICAL PARA BRASIL}

El Roadmap de Bioeconomía Tropical para Brasil preparado por el Agropolo Campinas-Brasil se concentra en los siguientes temas:

- Residuos agrícolas y urbanos

- Agricultura de precisión

- Aceites esenciales, aromáticos y plantas medicinales

- Sistemas de producción animal

- Uso sustentable del agua

- Las nuevas industrias del café y cítricos

- Ingredientes y alimentos funcionales

- Paquetes para alimentos y bebidas

- Tecnologías de proceso para alimentos y bebidas

- Biocombustibles avanzados y productos químicos de biomasa

- Enzimas y química verde. 
Estas áreas están agrupadas en macro-áreas de acuerdo con la Figura 2, presentada más adelante.

La idea básica es cambiar la forma de colaboración entre los investigadores de las instituciones públicas y acercarlos más a las demandas del sector privado. Un punto importante en este aspecto es que el foco de la investigación deja de ser multidisciplinario y pasa a ser interdisciplinario, con los esfuerzos sumados para solucionar problemas identificados en las cadenas productivas.

Figura 2 - Grandes áreas tratadas en el Proyecto de Bioeconomía.

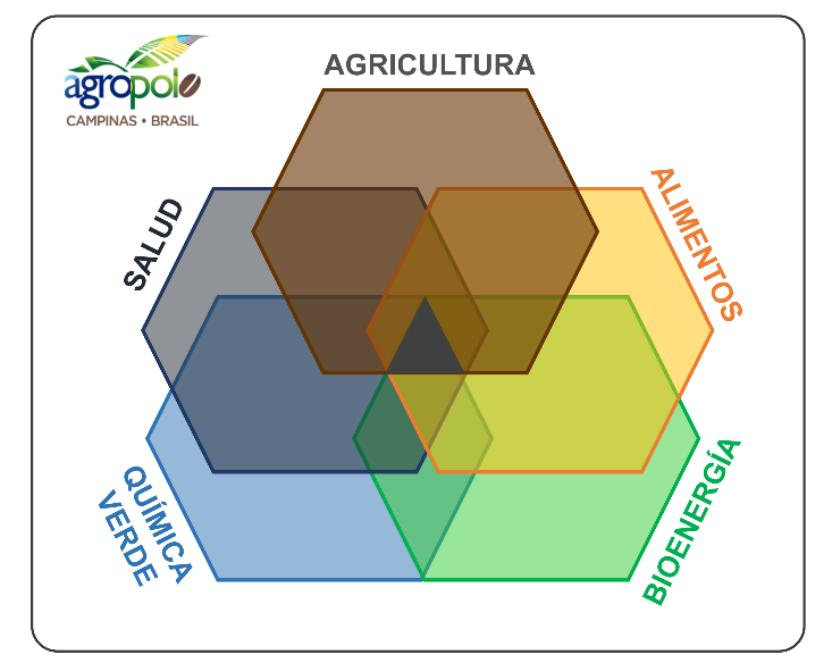

Desde punto de vista metodológico, los encuentros entre expertos de los sectores público y privado (Figura 3) fueron preparados previamente, procurando identificar las preguntas claves que debían ser discutidas, esperando encontrar respuestas y soluciones adecuadas para los problemas centrales de las cadenas productivas. La metodología utilizada en el proceso fue básicamente adaptada de otros rodmaps financiados por la FAPESP como el de bioetanol (CORTEZ, 2010). 
Figura 3 - Instituciones público y privadas de ciencia, tecnología e innovación de la región metropolitana de Campinas participantes del Agropolo Campinas-Brasil.

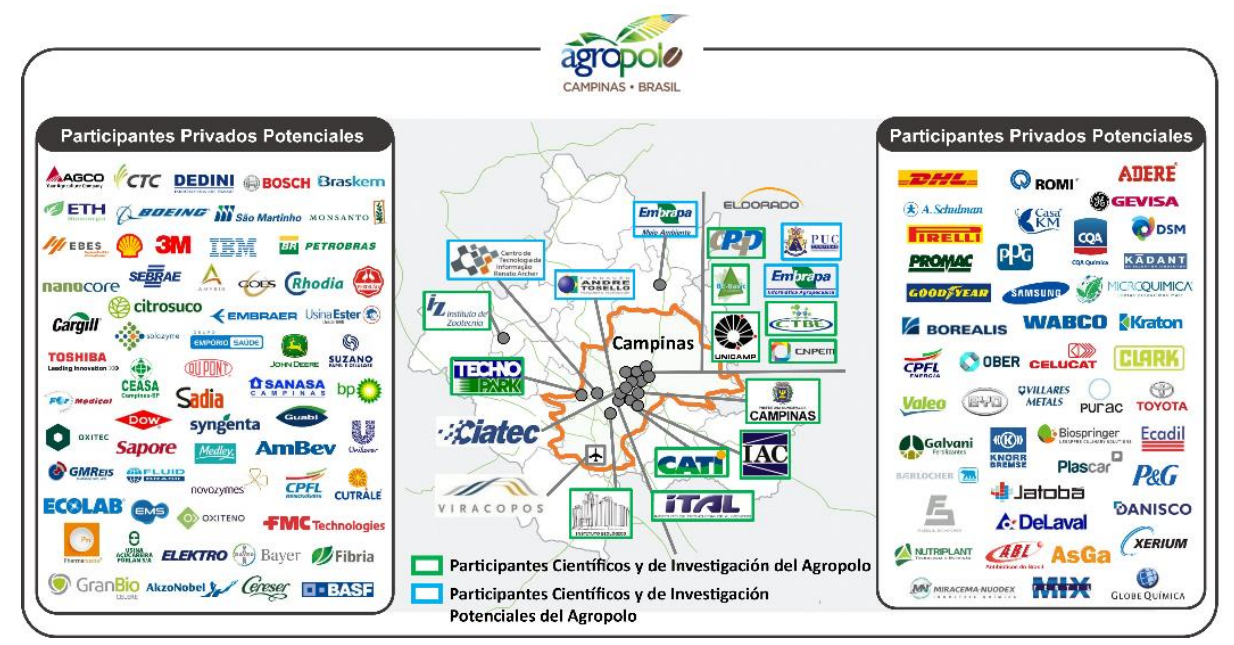

\section{RESULTADOS DEL PROYECTO PPPBio}

En la primera parte del proyecto PPPBio se llevaron a cabo discusiones para identificar las necesidades de investigación en las áreas de Agricultura, Alimentos/Salud, Química Verde/Bioenergía.

Como resultado, se identificaron por cada área estratégica, los factores limitantes (presentes y futuros), de cada una de ellas.

A continuación, se presenta un resumen de las propuestas de políticas públicas identificadas.

\section{CONCLUSIONES}

El objetivo de este artículo es presentar el Agropolo Campinas-Brasil, una plataforma de investigación interinstitucional creada em la región metropolitana de Campinas-Brasil para la valoración de los productos del agronegocio en el país.

Para este fin fue creado un primer proyecto en bioeconomía tropical con el apoyo financiero de la Fundación de Apoyo a la Investigación del Estado de São Paulo - FAPESP: el proyecto PPPBio, que también ha contado con apoyos diversos empresas públicas y privadas. El proyecto fue puesto en marcha, obteniéndose resultados importantes en la forma de un roadmap. 
El roadmap de bioeconomía tropical desarrollado en el proyecto de Políticas Públicas por el Agropolo Campinas-Brasil ha permitido:

- Establecer áreas estratégicas para el desarrollo regional en temas de bioeconomía tropical.

- Identificar incentivos para una plataforma de investigación e innovación multinstitucional enfocado en desarrollo socioeconómico regional.

- Apoyar los esfuerzos existentes a empresas creadas a partir de una base tecnológica.

- Establecer políticas públicas para mejorar las asociaciones entre los sectores público-privado (PPPs).

- Establecer programas de transferencia tecnológica y capacitación enfocadas en pequeñas y medias empresas.

- Mejorar la educación y capacitación de profesionales y técnicos en las áreas estratégicas de biotecnología, bioinformática, nanotecnología, emprendimiento, negocios, innovación, entre otras.

- Estimular la asociación con organismos internacionales enfocados en mercados estratégicos.

Tabla 1. Recomendaciones de políticas para el desarrollo de la bioeconomía en Brasil.

\begin{tabular}{|c|c|c|c|}
\hline \multirow[b]{2}{*}{ Tema } & \multicolumn{3}{|c|}{ Política Recomendada } \\
\hline & Corto Plazo & $\begin{array}{r}\text { Mediano } \\
\text { Plazo (2030) }\end{array}$ & $\begin{array}{l}\text { Largo Plazo } \\
\text { (2050) }\end{array}$ \\
\hline $\begin{array}{l}\text { Ciencia y } \\
\text { Tecnología }\end{array}$ & $\begin{array}{l}\text { - Implementar un } \\
\text { Plan Nacional } \\
\text { Estratégico } \\
\text { para } \\
\text { Bioeconomía. } \\
\text { Desarrollar un } \\
\text { Plan de } \\
\text { Comunicación } \\
\text { para mejorar la } \\
\text { concientización } \\
\text { de los } \\
\text { consumidores y } \\
\text { de los } \\
\text { mercados sobre } \\
\text { los beneficios } \\
\text { de } \\
\text { bioproductos. }\end{array}$ & $\begin{array}{l}\text { - Promocionar } \\
\text { los estudios } \\
\text { avanzados } \\
\text { para } \\
\text { asegurar y } \\
\text { monitorear el } \\
\text { impacto y las } \\
\text { ganancias de } \\
\text { la } \\
\text { bioeconomía } \\
\text { Mejorar el } \\
\text { impacto y la } \\
\text { calidad de la } \\
\text { C\&T en } \\
\text { bioeconomía. }\end{array}$ & $\begin{array}{l}\text { - Armonizar las } \\
\text { demandas por } \\
\text { tierra, comida, } \\
\text { ración, y } \\
\text { biocombustibles. }\end{array}$ \\
\hline $\begin{array}{l}\text { Educación y } \\
\text { Entrenamiento }\end{array}$ & $\begin{array}{ll}- & \text { Expandir la } \\
\text { capacitación } \\
\text { de los }\end{array}$ & $\begin{array}{l}\text { - } \quad \text { Reformular los } \\
\text { nivel superior }\end{array}$ & lanes de estudio de \\
\hline
\end{tabular}


C3-BIOECONOMY, Revista de Investigación y Transferencia en Bioeconomía Circular y Sostenible N² (2021)

\begin{tabular}{|c|c|c|c|}
\hline \multirow[b]{2}{*}{ Tema } & \multicolumn{3}{|c|}{ Política Recomendada } \\
\hline & Corto Plazo & $\begin{array}{r}\text { Mediano } \\
\text { Plazo (2030) }\end{array}$ & $\begin{array}{l}\text { Largo Plazo } \\
\text { (2050) }\end{array}$ \\
\hline & $\begin{array}{l}\text { investigadores } \\
\text { en temas } \\
\text { avanzados de } \\
\text { la } \\
\text { bioeconomía. } \\
\text { Introducir } \\
\text { nuevas } \\
\text { disciplinas } \\
\text { (nuevas áreas } \\
\text { tecnológicas) } \\
\text { en los currículos } \\
\text { de nivel } \\
\text { superior. } \\
\text { Desarrollar } \\
\text { cursos de } \\
\text { entrenamiento } \\
\text { orientados al } \\
\text { mercado. }\end{array}$ & $\begin{array}{ll}\text { - } & \text { Aumentar lo } \\
\text { investigador }\end{array}$ & $\begin{array}{l}\text { d de } \\
\text { diantes }\end{array}$ \\
\hline $\begin{array}{l}\text { Transferencia } \\
\text { Tecnológica y } \\
\text { Extensión }\end{array}$ & $\begin{array}{ll}\text { - } & \text { Implementar un } \\
\text { - } & \text { Aumsferencia te } \\
\text { pequeñas y me } \\
\text { alimentos para } \\
\text { número de los } p \\
\text { - } \quad \text { Implementar nu } \\
\text { licenciamiento } \\
\text { servicios entre e } \\
\text { jurídica y comp }\end{array}$ & $\begin{array}{l}\text { agenda regiono } \\
\text { lológica y de ex } \\
\text { :anismos y esfue } \\
\text { anas sobre todo } \\
\text { sustentabilidad, } \\
\text { ductos de alto } \\
\text { os modelos de } \\
\text { :ompartición de } \\
\text { ector Público y } \\
\text { bles con la Ley }\end{array}$ & $\begin{array}{l}\text { lal para } \\
\text { s empresas } \\
\text { icultura y } \\
\text { de empleos y } \\
\text { gado } \\
\text { ontratos de } \\
\text { productos y } \\
\text { on certeza } \\
\text { ción brasileña. }\end{array}$ \\
\hline $\begin{array}{c}\text { Innovación y } \\
\text { Emprendimiento }\end{array}$ & $\begin{array}{ll}\text { - } & \text { Incrementar la } \\
\text { - } & \text { Eliminar la buroc } \\
\text { - } & \text { Estimulariamiento } \\
& \text { pequeñas y me } \\
\text { - } & \text { Estimular las em } \\
& \text { mercados globo } \\
\text { - } & \text { Conectar y mej } \\
\end{array}$ & $\begin{array}{l}\text { ucación empres } \\
\text { xcia y facilitar el } \\
\text { ión tecnológica } \\
\text { anas } \\
\text { esas high-tech o } \\
\text { ar los ecosistemo }\end{array}$ & a los \\
\hline $\begin{array}{c}\text { Incentivos y } \\
\text { Financiamiento }\end{array}$ & 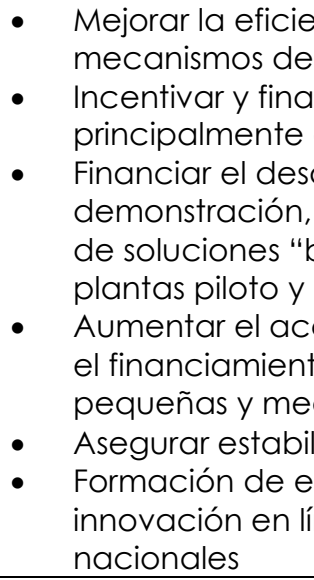 & $\begin{array}{l}\text { cia y crear nuev } \\
\text { centivos para I8 } \\
\text { ciar } \\
\text { sector privado. } \\
\text { ollo, } \\
\text { implementaciór } \\
\text { ", incluyendo } \\
\text { demonstración } \\
\text { so tecnológico } \\
\text { a las empresas } \\
\text { anas } \\
\text { ad del financian } \\
\text { sistemas colabc } \\
\text { a con las polític }\end{array}$ & $\begin{array}{l} \\
\\
\text { ialico para I\&D } \\
\text { ara la } \\
\text { iales } \\
\end{array}$ \\
\hline Regulación & $\begin{array}{ll}- & \text { Armonizar las pa } \\
\text { - } & \text { Para el desarrol } \\
& \text { para nuevas mo }\end{array}$ & $\begin{array}{l}\text { icas de tasas de } \\
\text { de productos "k } \\
\text { s más rápidos y } \\
\text { culas y nuevos } r\end{array}$ & $\begin{array}{l}\text { los incentivos } \\
\text { orincipalmente } \\
\text { y productos }\end{array}$ \\
\hline
\end{tabular}


Carbonell, S.A.M., Cortez, L.A.B., Madi, L.F.C., Anelafos, L.C., Baldassin Jr., R. \& Leal, R.L.V.

\begin{tabular}{|c|c|c|c|}
\hline \multirow[b]{2}{*}{ Tema } & \multicolumn{3}{|c|}{ Política Recomendada } \\
\hline & Corto Plazo & $\begin{array}{r}\text { Mediano } \\
\text { Plazo (2030) }\end{array}$ & $\begin{array}{l}\text { Largo Plazo } \\
\text { (2050) }\end{array}$ \\
\hline & \multicolumn{3}{|c|}{$\begin{array}{l}\text { Asegurar la sustentabilidad de los productos "bio": } \\
\text { recursos genéticos y exploración de la biodiversidad, } \\
\text { tracking y certificación de las materias primas y los } \\
\text { impactos ambientales }\end{array}$} \\
\hline $\begin{array}{c}\text { Colaboración } \\
\text { Global }\end{array}$ & \multicolumn{3}{|c|}{$\begin{array}{l}\text { - Mejorar la cooperación, el financiamiento y la buena } \\
\text { - } \quad \text { Implemanentar mecanismos para asegurar el cumplimiento } \\
\text { - } \quad \text { la transparencia global } \\
\text { - Armonizar los instrumentos regulatorios internacionales }\end{array}$} \\
\hline
\end{tabular}

Esta y otras iniciativas llevadas por los sectores público y privado en Brasil deberán crear las bases para un desarrollo más durable, generando productos de alta calidad y beneficiando a todas las cadenas productivas.

Con este objetivo, el Agropolo Campinas-Brasil considera la Red de Gestión de la Innovación en el Sector Agroalimentario (Red INNOVAGRO), como un apoyo estratégico en la implementación de sus acciones.

\section{AGRADECIMIENTOS}

Este trabajo fue financiado por la Fundación de Apoyo a la Investigación del Estado de São Paulo - FAPESP (Proyecto 2016 / 50198-0 - Agropolo CampinasBrasil: roadmap de las áreas estratégicas de investigación con el objetivo de crear un ecosistema de bioeconomía de clase mundial. La coordinación de Agropolo Campinas-Brasil también agradece a todos los demás socios y partes interesadas, principalmente a los coordinadores de los talleres de bioeconomía, ponentes, panelistas, patrocinadores, equipo de apoyo y participantes, involucrados en este proyecto.

Un agradecimiento especial a Martha Escalante y Raúl Rodríguez de la Red Innovagro por traducir este artículo al español.

\section{REFERENCIAS}

AGEMCAMP. Indicadores da RMC. Disponible en:

http://www.agemcamp.sp.gov.br/observatorio/indicadores.php

(última consulta en 18 de junio de 2021) 
BOUND, K. (2008). Brazil, The Natural knowledge economy, The Atlas of Ideas, Demos, London, SEl 2TU, UK, 159p., 2008.

CARBONELL, S.A.M., L.A.B. CORTEZ, L.F.C MADI, L.C. ANEFALOS, R. BALDASSIN JUNIOR, R.L.V. LEAL. (2021). Bioeconomy in Brazil: opportunities and guidelines for research and public policy for regional development. Biofuels, Bioproducts and Biorefining - Biofpr Journal, accepted for publication.

CARBONELL, S.A.M., L.A.B. CORTEZ, L.F.C MADI, L.C. ANEFALOS, R. BALDASSIN JUNIOR, R.L.V. LEAL. (2021). Bioeconomía Tropical, Roadmaps e Diretrizes para o Desenvolvimento da Bioeconomia no Brasil. ISBN 97865-994280-0-5, 163p.

CORTEZ, L.A.B. (Coord.), (2010). Sugarcane Bioethanol: R\&D for productivity and sustainability. ISBN 978-85-212-0530-2, Editora Edgard Blucher, $2010,992 p$.

CORTEZ, L. (2019). Políticas Públicas y Bioeconomía en Brasil. Presentación en el IX Encuentro de la Red Innovagro - Bioeconomía Circular y Ecosistemas de Innovación, Córdoba, España, junio 2019.

EUROPEAN COMMISSION. (2021). Roadmap for moving to a competitive low-carbon economy in 2050, disponible en: https://ec.europa.eu/clima/sites/default/files/2050_roadmap_en.pdf (última consulta en 18 de junio de 2021)

GERMAN FEDERAL MINISTRY OF EDUCATION AND RESEARCH, (2021). National Research Strategy Bioeconomy, our route towards biobased economy, 2030, 56p. Disponible en: http://biotech2030.ru/wpcontent/uploads/docs/int/bioeconomy 2030 germany.pdf (última consulta en 18 de junio de 2021)

SECRETARIA DE AGRICULTURA E ABASTECIMENTO DO ESTADO DE SÃO PAULO - APTA/IAC. (2012). INSTITUTO AGRONÔMICO (IAC) - "Orgulho nacional em terras paulistas" Campinas: Instituto Agronômico, 58 p. (Documentos IAC, 107) 
OECD. 2009). The Bioeconomy to 2030, designing a policy agenda, 321 p., 2009.

PREFEITURA MUNICIPAL DE CAMPINAS. (2015). Perfil do agronegócio no município de Campinas, 2015.

PREFEITURA MUNICIPAL DE CAMPINAS. (2015). Planejamento Estratégico de Ciência, Tecnologia e Inovação de Campinas (PECTI) 2015-2025.

FINNISH GOVERNMENT. (2014). THE FINNISH BIOECONOMY STRATEGY:

sustainable development from bioeconomy. 2014. 17p. Disponible en: https://biotalous.fi/wp-

content/uploads/2014/08/The Finnish Bioeconomy Strategy 1106201 41.pdf (última consulta en 18 de junio de 2021)

UK GOVERNMENT. (2021). Building a high value bioeconomy: opportunities from waste. 40p. disponible en:

https://assets.publishing.service.gov.uk/government/uploads/system/ uploads/attachment data/file/408940/BIS-15-

146_Bioeconomy_report_-_opportunities_from_waste.pdf lúltima consulta en 18 de junio de 2021)

US WHITE HOUSE. (2012). National Bioeconomy Blueprint. de Abril de 2012. disponible en: https://obamawhitehouse.archives.gov/sites/default/files/microsites/ ostp/national bioeconomy blueprint_april_2012.pdf (última consulta en 18 de junio de 2021) 